J. Med. 351:987-997.

9. Chan, J.L., et al. 2003. The role of falling leptin levels in the neuroendocrine and metabolic adaptation to short-term starvation in healthy men. J. Clin. Invest. 111:1409-1421.

10. Farooqi, I.S., et al. 2002. Beneficial effects of leptin on obesity, T cell hyporesponsiveness, and neuroendocrine/metabolic dysfunction of human congenital leptin deficiency. J. Clin. Invest. 110:1093-1103.

11. Oral, E.A., et al. 2002. Leptin-replacement therapy for lipodystrophy. N. Engl. J. Med. 346:570-578.

12. Oral, E.A., et al. 2002. Effect of leptin replacement on pituitary hormone regulation in patients with severe lipodystrophy. J. Clin. Endocrinol. Metab. 87:3110-3117

13. Rosenbaum, M., et al. 2002. Low dose leptin administration reverses effects of sustained weight-reduc- tion on energy expenditure and circulating concentrations of thyroid hormones. J. Clin. Endocrinol. Metab. 87:2391-2394.

14. Rosenbaum, M., et al. 2005. Low-dose leptin reverses skeletal muscle, autonomic, and neuroendocrine adaptations to maintenance of reduced weight. J. Clin. Invest. 115:3579-3586.

15. Farooqi, I.S., et al. 2007. Leptin regulates striatal regions and human eating behavior. Science. 317:1355.

16. Baicy, K., et al. 2007. Leptin replacement alters brain response to food cues in genetically leptindeficient adults. Proc. Natl. Acad. Sci. U. S. A. 104:18276-18279.

17. Boozer, C.N., et al. 2001. Synergy of sibutramine and low-dose leptin in treatment of diet-induced obesity in rats. Metabolism. 50:889-893.
18. Farooqi, I.S., et al. 2001. Partial leptin deficiency and human adiposity. Nature. 414:34-35.

19. Begriche, K., et al. 2008. Partial leptin deficiency favors diet-induced obesity and related metabolic disorders in mice. Am. J. Physiol. Endocrinol. Metab. 294:E939-E951.

20. Verdich, C., et al. 2001. Leptin levels are associated with fat oxidation and dietary-induced weight loss in obesity. Obes. Res. 9:452-461.

21. Filozof, C.M., et al. 2000. Low plasma leptin concentration and low rates of fat oxidation in weightstable post-obese subjects. Obes. Res. 8:205-210.

22. Batterham, R.L., et al. 2007. PYY modulation of cortical and hypothalamic brain areas predicts feeding behaviour in humans. Nature. 450:106-109.

23. [No authors listed]. 2007. Gastroenterology. 132(Issue 6), cover figure.

\title{
How irritating: the role of TRPA1 in sensing cigarette smoke and aerogenic oxidants in the airways
}

\author{
Sidney A. Simon ${ }^{1}$ and Wolfgang Liedtke ${ }^{1,2}$
}

1Department of Neurobiology and 2Department of Medicine, Division of Neurology, Duke University Medical Center, Durham, North Carolina, USA.

\begin{abstract}
Airway irritants cause a variety of lung pathologies. Two separate studies, the first recently reported in the JCI by Bessac et al. and the second reported by Andrè et al. in the current issue of the JCI (see the related article beginning on page 2574), have identified irritants that activate transient receptor potential cation channel, subfamily A, member 1 (TRPA1) receptors in airway sensory neurons, resulting in neurogenic inflammation and respiratory hypersensitivity. The identification of TRPA1 activation by toxicants from cigarette smoke and polluted air, such as crotonaldehyde, acrolein, and oxidizing agents such as hydrogen peroxide, is an important finding. These two studies enhance our understanding of how pollution and cigarette smoke can damage airway function and will hopefully pave the way for the development of rational alternative therapeutics for such airway injury.
\end{abstract}

Inhalation of pollutants is associated with adverse cardiovascular and respiratory diseases and may lead to an increase in mortality. In addition, persons with sensitized airways often display respiratory hypersensitivity to many chemical irritants found in polluted air. Two critical issues in understanding the etiology of these pathologies are the identification of the responsible chemicals and the iden-

Nonstandard abbreviations used: CSE, cigarette smoke aqueous extract; DRG, dorsal root ganglia; TRPA1, transient receptor potential cation channel subfamily A, member 1; TRPV1, transient receptor potential cation channel, subfamily V, member 1 .

Conflict of interest: The authors receive financial support from Philip Morris USA and Philip Morris International.

Citation for this article: J. Clin. Invest. 118:2383-2386 (2008). doi:10.1172/JCI36111. tification of their receptors on epithelial cells and on sensory neurons that innervate these cells.

\section{Anatomy of airways and transient receptor potential channels}

The airways are innervated by branches of the trigeminal and vagal nerves (Figure 1). Among the many classes of nerve fibers are the polymodal nociceptors (PMNs). These unmyelinated neurons send signals that cause the perception of pain in response to potentially damaging thermal, mechani$\mathrm{cal}$, and chemical stimuli. Their activation induces protective reflexes and nocifensive behaviors (defensive behavior that is elicited by sensory stimuli that have the potential to cause injury) that include apnea, bradycardia, coughing, mucus secretion, and avoidance behavior. Some well established chemical irritants that activate PMNs include capsaicin (the pungent compound in chili pepper and Mace brand defense sprays), allyl isothiocyanate (present in mustard, wasabi, and horseradish), formaldehyde, nicotine, acid, hydrogen peroxide $\left(\mathrm{H}_{2} \mathrm{O}_{2}\right)$, chlorine, acrolein, and, finally, smoke generated from tobacco. The latter differs from the other compounds in that it comprises at least 5,000 distinct chemicals at varying concentrations.

Although there are many types of PMNs, the most common are those that are activated by capsaicin through its receptor, transient receptor potential cation channel, subfamily V, member 1 (TRPV1). TRPV1 is a member of the TRPV subfamily of ion channels that are all inhibited by the polyvalent cationic dye and ion channel blocker ruthenium red, but specific antagonists may exist for individual transient receptor potential channels. For TRPV1, one such antagonist is capsazepine, a synthetic analogue of capsaicin. When capsaicin-sensitive neurons are activated, they transmit nociceptive information to upstream relay centers within the CNS that are associated with pain perception and, importantly, these neurons also release proinflammatory mediators (1). With respect to cigarette smoke aqueous extract (CSE) as a stimulus, capsaicin-sensitive nociceptors appear to have an important role in physiological 


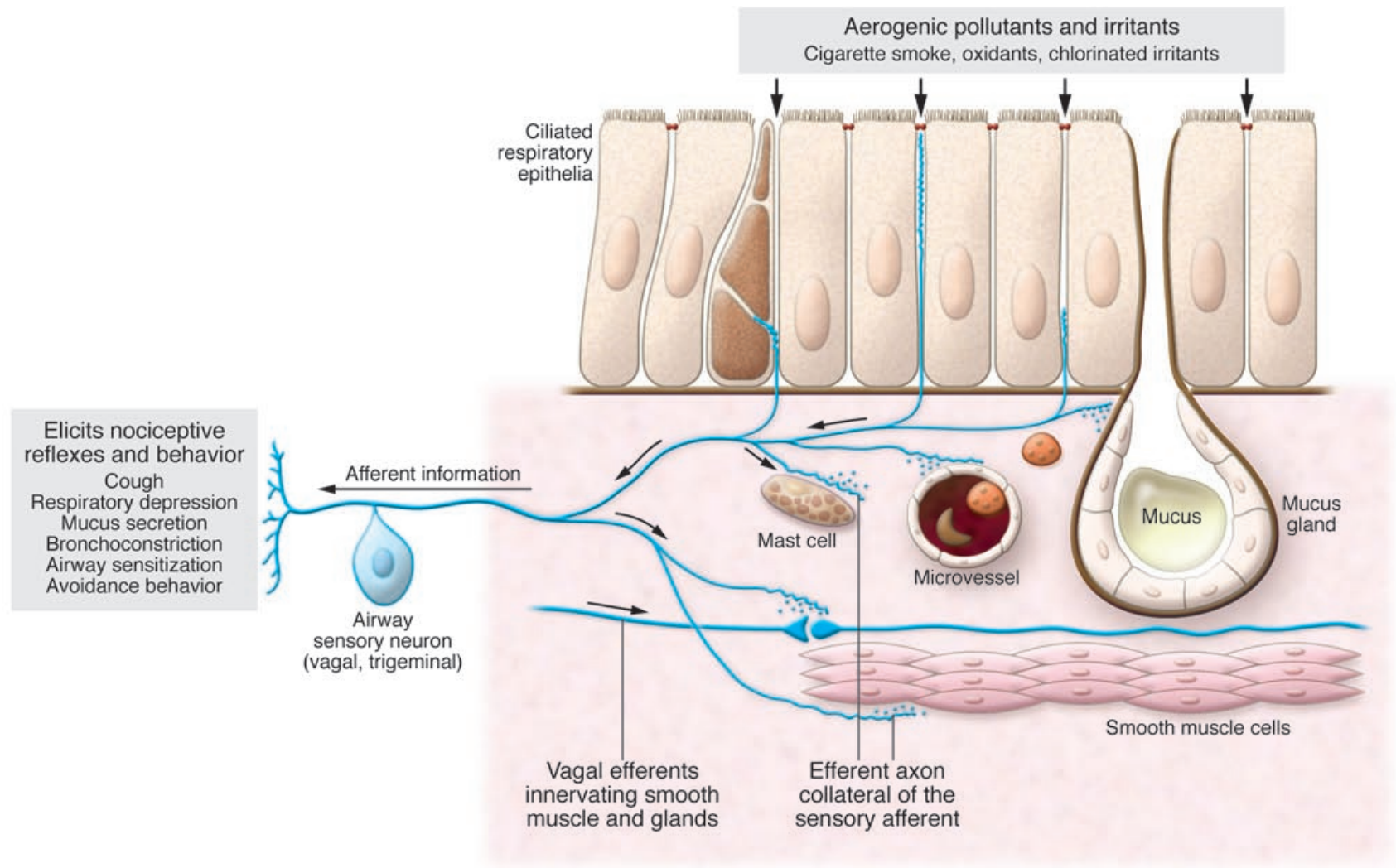

Figure 1

A schematic overview of the airway-sensory neuron unit. Sensory neurons, with their soma located in vagal (nodose or jugular) or trigeminal ganglia, extend processes into the submucous layer, reaching even into the single-cell layer of ciliated airway epithelial cells. Collaterals of the afferent axons innervate mast cells, capillaries, smooth muscle cells, and exocrine glands. Exposure to airway irritants can elicit nociceptive reflexes via activation of airway nociceptive sensory neurons and their afferent inputs being processed via synaptic relay in the brainstem. These protective reflexes can lead to coughing, respiratory depression until respiratory arrest, mucus hypersecretion, bronchial hyperresponsiveness, and avoidance behavior. Axonal collaterals can mediate the same consequences, without synaptic relay, via secretion of the neuropeptides bradykinin and calcitonin gene-related peptide, a process that is also referred to as neurogenic inflammation. Adapted with permission from Taylor \& Francis (26).

changes in airways and afferent control of respiration in response to CSE. Specifically, in rodent neonates, capsaicin pretreatment has been shown to induce degeneration of respiratory tract nociceptors and a longlasting desensitization of the airways to cigarette smoke (2). In this regard, in rat airways, it was found that capsaicin pretreatment prevented plasma extravasation (a critical component of the inflammatory response that results from the activation of sensory nerve endings and the subsequent release of proinflammatory neuropeptides) in response to exposure to cigarette smoke (2). Interestingly, plasma extravasation was inhibited by ruthenium red but not by capsazepine (3). These results indicated that TRPV1 capsaicin receptor-expressing neurons are important in airway sensitivity but that the TRPV1 capsaicin receptor is not the receptor for the majority of chemicals in cigarette smoke.

\section{TRPA1 and cigarette smoke}

In the current issue of the JCI, the report by Andrè et al. (4) identifies the $\mathrm{Ca}^{2+}$ permeable transient receptor potential cation channel, subfamily A, member 1 (TRPA1) channel (Figure 2) as the receptor for some of the principal components of CSE, namely crotonaldehyde and acrolein (4). TRPA1, like TRPV1, is expressed by trigeminal and nodose/jugular ganglia neurons and moreover, both channels are most often found in the same neuron $(5,6)$. This means that activation of TRPA1 will likely exert effects similar to those observed following the activation of TRPV1. In addition, these channels are generally, but not always, activated by different agonists (an exception is allicin, the pungent ingredient in garlic, which activates both TRPA1 and TRPV1 receptors; refs. 7,8$)$. Specifically, TRPA1 is exclusively activated by mustard oil, cinnamaldehyde (a compound found in cinnamon), lipids (9), chlorine (see below), the pollutant acrolein (10-14), as well as endogenous aldehydes (15). TRPA1 is not inhibited by capsazepine but rather by a specific antagonist, HC-030031 (6).

Using a variety of techniques, Andrè et al. (4) showed that the $\alpha, \beta$-unsaturated aldehydes crotonaldehyde and acrolein, the most abundant unsaturated aldehydes in CSE, induce neurogenic inflammation by stimulating TRPA1 channels coexpressed with TRPV1 on capsaicin-sensitive nociceptors. After identifying TRPA1 neurons in guinea pig jugular ganglia, the authors cultured these neurons and used $\mathrm{Ca}^{2+}$ imaging to show that, in capsaicin-sensitive neurons, CSE and both unsaturated aldehydes activated these neurons in a dose-dependent manner. Importantly, the responses were inhibited by HC-030031, but not by capsazepine or a variety of ROS scavengers. They showed that nicotine 


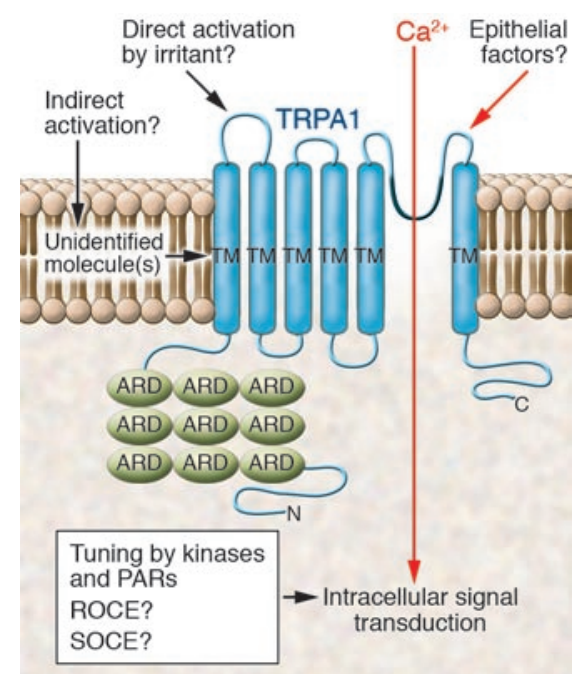

Airway sensory neuron

\section{Figure 2}

The TRPA1 channel is expressed on sensory nerve endings, and $\mathrm{Ca}^{2+}$ permeates the channel in response to airway irritants. The studies by Andrè et al. (4) and Bessac et al. (16) unequivocally establish the necessity of the gene product of the Trpa1 gene, the TRPA1 channel, for the response of airway sensory neurons to airborne irritants. However, based on this important step forward, it will be interesting to study pertinent follow-up questions regarding the signaling hierarchy, which, as we now know, contains Trpa1 gene products as one critical hub. For example, do airborne irritants activate TRPA1 directly or indirectly? As to the latter, this can possibly be achieved via other sensory neuron surface receptors or via interaction of the irritants with (lipid) components of the outer cell membrane. Moreover, what role do epithelial factors play in the activation of TRPA1-positive airway sensory neurons? In terms of intracellular signal transduction in these neurons, it will be interesting to learn how proteinase-activated receptors (PARs) and kinases sensitize (or desensitize) TRPA1, and what eventual roles store-operated $\mathrm{Ca}^{2+}$ entry (SOCE) and receptor-operated $\mathrm{Ca}^{2+}$ entry (ROCE) play in $\mathrm{Ca}^{2+}$ influx via TRPA1 (24). In the figure, the channel is depicted with its numerous $\mathrm{N}$-terminal ankyrin-repeat domains (ARDs), which supposedly anchor the channel to the cytoskeleton; also illustrated is the channel's pore-loop domain (highlighted in dark blue) between transmembrane (TM) domains 5 and 6. C, C-terminal; N, N-terminal. Adapted with permission from Cell (27). did not activate TRPA1 and that acetaldehyde, the most abundant saturated aldehyde present in CSE, produced a small response at high concentrations but was not inhibited by HC-030031. Also, they provide evidence that HEK293 cells heterologously expressing TRPA1 were responsive to CSE as well as to the two unsaturated aldehydes. This was true also for dorsal root ganglia (DRG) neurons from Trpa $1^{+/+}$but not from Trpa1 $1^{-/-}$mice. Using slices of guinea pig airways, they showed that acrolein or crotonaldehyde induced release of the neuropeptides substance $P$ and calcitonin gene-related peptide. This release was reduced when the neurons were desensitized with capsaicin and/or when extracellular $\mathrm{Ca}^{2+}$ was removed, thus showing that CSE and unsaturated aldehydes cause an extracellular $\mathrm{Ca}^{2+}$-dependent release of neuropeptides from capsaicinsensitive airway sensory nerve terminals. In physiological experiments using isolated guinea pig bronchial rings, the authors showed that CSE, as well as acrolein or crotonaldehyde, produced a contraction of the bronchial rings that was inhibited by HC-030031 but not by capsazepine or ROS scavengers. Finally, installation of CSE into the trachea of wild-type and Trpa1 $1^{-/-}$mice revealed that plasma extravasation was only observed in wild-type mice. In summary, Andrè et al. showed that cigarette smoke-induced airway neurogenic inflammation is mediated by $\alpha, \beta$-unsaturated aldehydes and their activation of the TRPA1 receptor.

\section{TRPA1 and oxidizing agents}

A related, recently published JCI article by Bessac et al. (16) made use of the fact that airway nerve terminals are activated and sensitized by oxidizing chemicals, including $\mathrm{H}_{2} \mathrm{O}_{2}$, ozone, and chlorine (16). Oxidant activation of airway sensory neurons can induce respiratory depression, nasal obstruction, sneezing, cough, and pain. Previous studies have demonstrated that destruction of capsaicin-sensitive neurons eliminates chlorine- and $\mathrm{H}_{2} \mathrm{O}_{2}$-induced airway reflexes (17), and several recent studies indicated that TRPA 1 is activated by $\mathrm{H}_{2} \mathrm{O}_{2}$ $(18,19)$. Specifically, Andersson et al. (19) showed in DRG neurons that $\mathrm{H}_{2} \mathrm{O}_{2}$ exposure results in a delayed $\mathrm{Ca}^{2+}$ response that was absent in DRG neurons from Trpa1 $1^{-/}$ mice. In their recent JCI study, Bessac et al. (16) showed similar effects in capsaicin- and mustard oil-sensitive mouse trigeminal and nodose ganglia neurons. Moreover, they also demonstrated that TRPA 1 is also activated by hypochlorite $\left(\mathrm{OCl}^{-}\right)$and chlorine. They further verified the effects of these oxidizing agents in HEK293 cells heterologously expressing TRPA1 channels. They went on to show that only in wild-type but not in Trpa1 $1^{-/-}$mice did these agents produce a marked effect both in respiratory physiological parameters such as breathing frequency and tidal volume. Moreover, DRG-mediated nocifensive behavior was significantly attenuated in Trpa $1^{-/-}$mice after hind paw injection of $\mathrm{H}_{2} \mathrm{O}_{2}$, also indicating a dependency on TRPA1 in the mediation of acute pain responses to $\mathrm{H}_{2} \mathrm{O}_{2}$.

\section{Summary}

These studies invite many meaningful and compelling questions (Figure 2). For example, which downstream pathways are operative when TRPA 1 is activated that may lead to hypersensitivity; what other proinflammatory compounds are released; and what is the role of airway epithelial cells in the modulation of the relevant nociceptors $(6,20-25)$ ? Nevertheless, much progress has been made as both of these studies $(4,16)$ point to a role for TRPA1 channels in response to pollutants, whether they originate from cigarette smoke, oxidizing agents, or from other irritants in the air. It is evident from these studies that we should consider topical application of specific TRPA1 antagonists to airways, with the potential to benefit humans exposed to pollutants; in particular, those subjects with manifest hypersensitivities should be considered, such as those effected by widespread respiratory diseases such as chronic obstructive pulmonary disease, emphysema, and chronic asthma. Worldwide, as more than several hundred million humans are exposed to severe air pollution, and a significant fraction of them also smoke tobacco, another highly relevant issue is that of human susceptibility factors that predispose subjects to a particularly severe (or mild) response to air pollution with or without exposure to cigarette smoke. Now that the TRPA1 gene has been placed in the limelight of this pathophysiology, this question can be addressed in a more specific manner. 
Address correspondence to: Sidney A. Simon, Department of Neurobiology, Duke University Medical Center, 327C Bryan Research Building, Box 3209, Durham, North Carolina 27708, USA. Phone: (919) 684-4178; Fax: (919) 684-4431; E-mail: sas@neuro.duke.edu.

1. Cechetto, D.F., Standaert, D.G., and Saper, C.B. 1985. Spinal and trigeminal projections to the parabarchial nucleus of the rat. J. Comp. Neurol. 240:153-160.

2. Lundberg, J.M., and Saria, A. 1983. Capsaicininduced desensitization of airway mucosa to cigarette smoke, mechanical and chemical irritants. Nature. 302:251-253.

3. Geppetti, P., et al. 1993. Ruthenium red. Br. J. Pharmacol. 108:646-650.

4. Andrè, E., et al. 2008. Cigarette smoke-induced neurogenic inflammation is mediated by $\alpha, \beta$ unsaturated aldehydes and the TRPA1 receptor in rodents. J. Clin. Invest. 118:2574-2582.

5. Nassenstein, C., et al. 2008. Expression and function of the ion channel TRPA1 in vagal afferent nerves innervating mouse lungs. J. Physiol. (Lond.) 586:1595-1604.

6. Nassenstein, C., et al. 2008. Expression and function of the ion channel TRPA1 in vagal afferent nerves innervating mouse lungs. J. Physiol. 586:1595-1604.

7. Salazar, H., et al. 2008. A single N-terminal cysteine in TRPV1 determines activation by pungent compounds from onion and garlic. Nat. Neurosci. 11:255-261.

8. Macpherson, L.J., et al. 2005. The pungency of garlic: activation of TRPA 1 and TRPV1 in response to allicin. Curr. Biol. 15:929-934.

9. Maher, M., et al. 2008. Activation of TRPA1 by farnesyl thiosalicylic acid. Mol. Pharmacol. 73:1225-1234.

10. Hinman, A., Chuang, H., Bauitsta, D.M., and Julius, D. 2006. TRP channel activation by reversible covalent modification. Proc. Natl. Acad. Sci. U. S. A. 103:19564-19568.

11. Calixto, J.B., Kassuya, C.A.L., André, E., and Ferreira, J. 2005. Contribution of natural products to the discovery of the transient receptor potential (TRP) channels family and their functions. Pharmacol. Ther. 106:179-208.

12. Bautista, D.M., et al. 2006. TRPA1 mediates the inflammatory actions of environmental irritants and proalgesic agents. Cell. 124:1269-1282.

13. Niforatos, W., et al. 2007. Activation of TRPA1 channels by the fatty acid amide hydrolase inhibitor 3'-carbamoylbiphenyl-3-yl cyclohexylcarbamate (URB597). Mol. Pharmacol. 71:1209-1216.

14. Merrill, A.W., Cuellar, J.M., Judd, J.H., Carstens, M.I., and Carstens, E. 2008. Effects of TRPA1 agonists mustard oil and cinnamaldehyde on lumbar spinal wide-dynamic range neuronal responses to innocuous and noxious cutaneous stimuli in rats. J. Neurophysiol. 99:415-425.

15. Trevisani, M., et al. 2007. 4-Hydroxynonenal, an endogenous aldehyde, causes pain and neurogenic inflammation through activation of the irritant receptor TRPA1. Proc. Natl. Acad. Sci. U. S. A. 104:13519-13524.

16. Bessac, B.F., et al. 2008. TRPA1 is a major oxidant sensor in murine airway sensory neurons. J. Clin. Invest. 118:1899-1910.

17. Morris, J.B., Wilkie, W.S., and Shusterman, D.J. 2005. Acute respiratory responses of the mouse to chlorine. Toxicol. Sci. 83:380-387.

18. Sawada, Y., Hosokawa, H., Matsumura, K., and Kobayashi, S. 2008. Activation of transient recep- tor potential ankyrin 1 by hydrogen peroxide. Eur. J. Neurosci. 27:1131-1142.

19. Andersson, D.A., Gentry, C., Moss, S., and Bevan, S. 2008. Transient receptor potential A1 is a sensory receptor for multiple products of oxidative stress. J. Neurosci. 28:2485-2494.

20. Mizushima, T., et al. 2006. Noxious cold stimulation induces mitogen-activated protein kinase activation in transient receptor potential (TRP) channels TRPA1- and TRPM8-containing small sensory neurons. Neuroscience. 140:1337-1348.

21. Oortgiesen, M., Veronesi, B., Eichenbaum, G., Kiser, P.F., and Simon, S.A. 2000. Residual oil fly ash and charged polymers activate epithelial cells and nociceptive sensory neurons. Am. J. Physiol. Lung Cell Mol. Physiol. 278:L683-L695.

22. Bandell, M., et al. 2004. Noxious cold ion channel TRPA 1 is activated by pungent compounds and bradykinin. Neuron. 41:849-857.

23. Dai, Y., et al. 2007. Sensitization of TRPA1 by PAR2 contributes to the sensation of inflammatory pain. J. Clin. Invest. 117:1979-1987.

24. Wang, S., et al. 2008. Phospholipase C and protein kinase A mediate bradykinin sensitization of TRPA1: a molecular mechanism of inflammatory pain. Brain. 131:1241-1251.

25. Taylor-Clark, T.E., et al. 2008. Prostaglandininduced activation of nociceptive neurons via direct interaction with transient receptor potential A1 (TRPA1). Mol. Pharmacol. 73:274-281.

26. Lee, L.Y., and Undem, B.J. 2005. Bronchopulmonary vagal afferent nerves. In Advances in vagal afferent neurobiology. B.J. Undem and D. Weinreich, editors. CRC Press/Taylor \& Francis. Boca-Raton, Florida, USA. 279-313.

27. Liedtke, W., et al. 2000. Vanilloid receptor-related osmotically activated channel (VR-OAC), a candidate vertebrate osmoreceptor. Cell. 103:525-535.

\title{
Measles virus breaks through epithelial cell barriers to achieve transmission
}

\section{Makoto Takeda}

\author{
Department of Virology, Faculty of Medicine, Kyushu University, Fukuoka, Japan.
}

\begin{abstract}
Measles is a highly contagious disease that causes immunosuppression in patients. Measles virus infection has been thought to begin in the respiratory epithelium and then spread to lymphoid tissue. In this issue of the JCI, Leonard et al. provide data to suggest an alternative model of measles virus pathogenesis (see the related article beginning on page 2448). In human primary epithelial cells and rhesus monkeys in vivo, the authors show that initial infection of respiratory epithelium is not necessary for the virus to enter the host but that viral entry into epithelial cells via interaction of the virus with a receptor located on the basolateral side of the epithelium is required for viral shedding into the airway and subsequent transmission.
\end{abstract}

Measles is an acute, viral infectious disease characterized by high fever, cough,

Nonstandard abbreviations used: CDV, canine distemper virus; $\mathrm{F}$, fusion (protein); $\mathrm{H}$, hemagglutinin (protein); MV, measles virus; SLAM, signaling lymphocytic activation molecule.

Conflict of interest: The author has declared that no conflict of interest exists.

Citation for this article: J. Clin. Invest. 118:2386-2389 (2008). doi:10.1172/JCI36251. and a maculopapular rash. Measles causes temporary and severe immunosuppression in patients, and secondary bacterial infections are a major cause of measles-related deaths (1). Studies have clearly shown that measles virus (MV), the causative agent, mainly replicates in lymphoid organs and causes devastating damage to the immune system of infected individuals (1). Despite the availability of effective vaccines, measles is still responsible for about $4 \%$ of deaths among children under 5 years of age worldwide (2). Importantly, the disease is transmitted via respiratory aerosols and is highly contagious in nature (1). In this issue of the JCI, Leonard et al. (3) report their detailed analyses of the interaction of MV with human host cells, in addition to data regarding MV pathogenesis in a rhesus monkey model. Their studies have revealed an elegant strategy by which MV propagates in patients and is transmitted to other target individuals and provide a good explanation for the highly contagious nature of measles.

\section{MV targets the host immune system}

$\mathrm{MV}$ is an enveloped virus possessing a nonsegmented negative-strand RNA genome and is classified into the Morbillivirus genus 